\title{
5G Internet of Radio Light Virtual Reality System
}

\author{
Ben Meunier, John Cosmas \\ WNCC, School of Engineering and Design \\ Brunel University London \\ Uxbridge, Middlesex, UB8 3PH, UK \\ E-mail: \{1408747@brunel.ac.uk,john.cosmas@brunel.ac.uk\}
}

\begin{abstract}
Virtual Reality (VR) is a technology that is rapidly developing, leading to a whole array of innovative commercially viable products. Some of the challenges facing the early development of Virtual Reality (VR) and Augmented Reality (AR) include high cost, restricted physical movement and laborious setup. This paper highlights several of these challenges and outlines an architecture in which systems can require less specialised equipment, be used with greater freedom and are simpler to setup. This paper shows how using the correct applications, the Internet of Radio Light (IoRL) architecture could lead to enhanced VR experiences. Specifically, a wireless six Degrees of Freedom (DOF) VR system for both existing mobile and PC operated VR. The aim is, to exploit the existing IoRL architecture to provide a safer, wireless, high speed, less laborious, more immersive and improved VR experience with broader applications.
\end{abstract}

Keywords - Virtual Reality (VR); Augmented reality (AR); (IoRL); Degrees of Freedom (DOF);

\section{INTRODUCTION $($ IORL $)$}

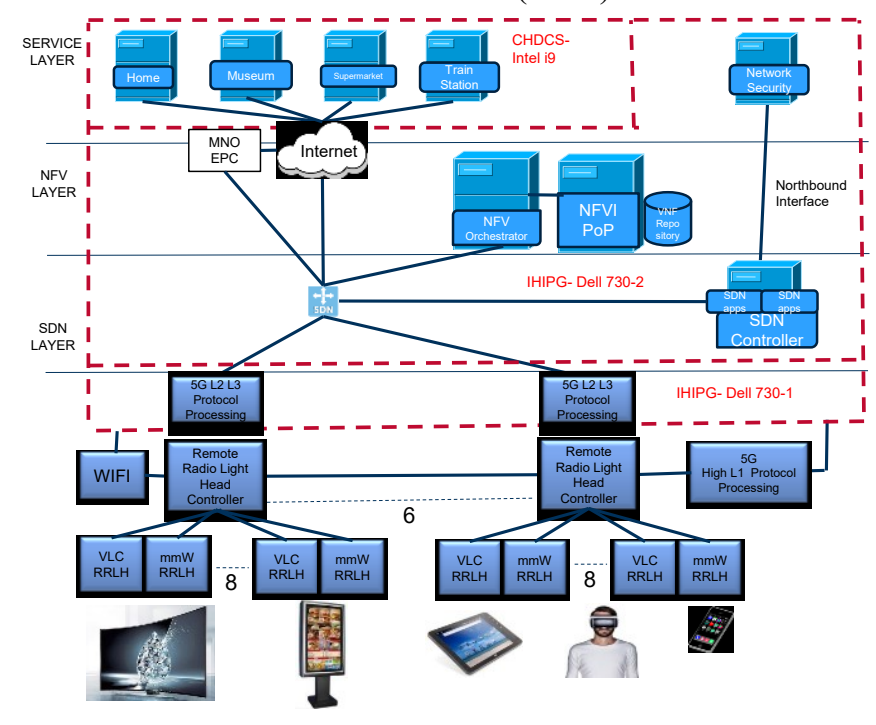

Fig.1. IoRL architecture

The IoRL [1][2] architecture shown in Fig.1, is designed to provide $5 \mathrm{G}$ networking within buildings, delivering greater than $10 \mathrm{Gbs}$ data rates with a latency under $1 \mathrm{~ms}$ as well as location sensing services with sub $10 \mathrm{~cm}$ accuracy. This is made possible by modulating existing lighting infrastructure for Visible Light Communications (VLC) and using millimetre wave (mmWave) transmission to make Remote Radio Light Heads (RRLH). These light heads act as an array of high-speed transmitters, to replace a singular $\mathrm{Wi}-\mathrm{Fi}$ radio-transmission router commonly found in buildings. The RRLH as shown in Fig. 2 houses both the mmWave and VLC transceivers discreetly within an existing light rose, acting as a frontend for the IoRL system.

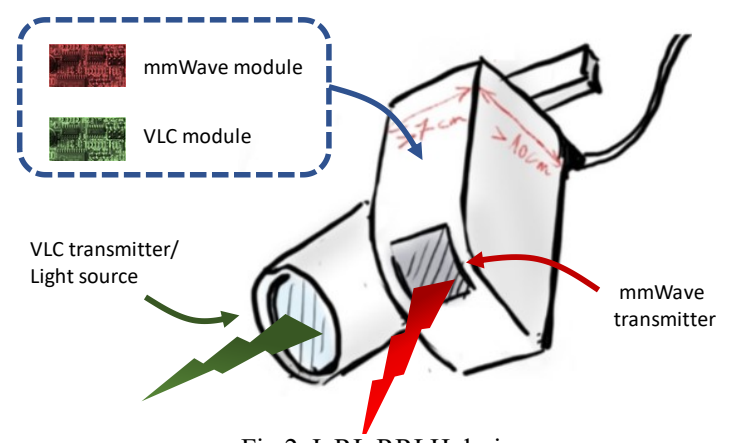

Fig.2. IoRL RRLH design

The IoRL project has the advantage of combining VLC and mmWave transmission in close proximity to users and user equipment (UE), thus reducing the distance between transmitters and UE, thereby reducing latency and increasing data rates. Furthermore, light fittings are regularly located in raised, central positions, which provide ideal data coverage. Buildings often contain multiple light fittings in a single room, increasing coverage, decreasing distance and allowing for location sensing of UEs. At the UE terminal, an 'IoRL dongle' is required to transmit and receive data between the RRLH transceivers and the UE. The dongle itself contains the mmWave and VLC transceivers along with a processor and adjustable interface for the required UE.

Indoor location sensing (IPS) is currently restricted to accuracies of a few metres, using Global Positioning Systems (GPS) [3] and Wireless Local Area Network fingerprint techniques [4]. The IoRL project will use VLC and mmWave transmission for location sensing to provide accuracies below $10 \mathrm{~cm}$. The IPS will require the IoRL RRLH controller to use $5 \mathrm{G}$ protocols to obtain the Round Trip Time Difference of Arrival (TDoA), which it will measure using a $0.1 \mathrm{~ns}$ clock derived from the 4 ns system clock. The UE will also measure the Received Signal Strength for the VLC and mmWave transmissions. This data is processed by an application within the Software Defined Network (SDN) that computes the UE 
location. Received Signal Strength (RSS) algorithm simulations have already provided insight into possible sub $2 \mathrm{~cm}$ accuracies using VLC alone, however this technology is still in the developmental stage [5][6][7]. In these simulations, four VLC transmitters tracked a single object in a $3 \mathrm{~m} \times 3 \mathrm{~m} \times 3 \mathrm{~m}$ area.

\section{ViRTUAL REALity SyStemS}

Virtual reality (VR) systems are becoming more immersive and opening up new exciting ways to experience, educate and entertain. The main objective of VR systems is to provide users with a greater sense of immersion in a virtual world. There are a number of immersion factors [8] that contribute to this user experience. Arguably, the greatest of these factors is the freedom of movement within a virtual environment, making wireless VR systems of great interest. This is evident from the rise in wireless VR systems such as Oculus project Santa Cruz [9] and the Vive Focus [10]. VR has developed an increasing number of applications from military training, scientific visualisation, and remote surgery to healthcare [11], all of which could benefit from the wireless freedom to allow enhanced user physical involvement. In order for VR to provide an impressive and comfortable user experience, high data rates and low latency are critical. Typical 4G networks place limitations on VR applications and cannot provide the bandwidth required. $5 \mathrm{G}$ networks however offer a substantial increase in latency and bandwidth, which will improve existing experiences and facilitate innovative ones. [12].

At present, there are three main VR systems; mobile operated VR (MOVR), which uses a mobile phone, held in a headset, PC operated VR (PCOVR), which uses computer processing and external tracking, and Standalone VR (SaVR) which requires only a HMD and no external components. SaVR works differently and does not fit within the scope of this paper, however it is valuable to use for comparison. PCOVR is substantially superior to MOVR due to its higher resolution, faster processing and capacity to track user movement, in general delivering a greater sense of immersion. Tracking a user permits for more DOF [13] [14] within a virtual world. MOVR systems are limited at 3DOF whilst most PCOVR deliver 6DOF. Sensors within a mobile or Head Mounted Display (HMD) can only provide 3DOF from rotational movement (yaw, pitch and roll). Location data provides an additional three DOF from translational movement in $\mathrm{x}, \mathrm{y}$ and $\mathrm{z}$ planes (surge, heave and sway). Current new VR systems leave substantial room for improvement. This proposal aims to eliminate the following factors:

- The restricted level of immersion of MOVR systems. Limited to 3DOF, through lack of external tracking systems capable of monitoring user movement.

- The expensive, laborious to install, external trackers of PCOVR. The positioning and arrangement of which requires specific setup criteria and can only be used by a single Operating System (OS).

- The number of cables necessary to connect the HMD to the PC in current PCOVR systems. These can be a trip hazard while additionally limiting the area a user can travel. The combination of the trackers, fixed tracking area and cabled HMD means users are restricted by an approximate $4 \mathrm{mx} 4 \mathrm{~m}$ physical area [15] thereby not able explore a virtual world fully.

\section{SOLUTION}

Integrating the IPS proposed by the IoRL project for VR, would make it possible to track the VR user's movements, eliminating the need for external trackers in addition to providing MOVR systems with 6DOF capabilities. The high data rates and wireless transmission of the IoRL project could also eliminate the need for cables, providing wireless VR.

\section{A. PCOVR}

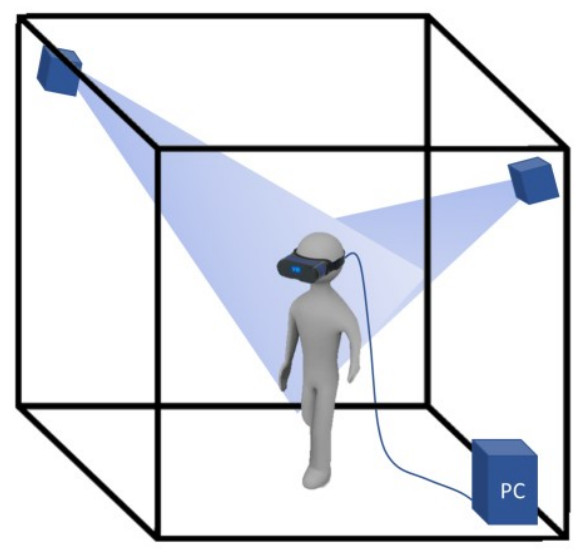

Fig. 3. General PCOVR configuration

Fig. 3 illustrates the general setup of the common HTC Vive [16] VR system. The two Base station trackers, located in the corners of the playable area, scan the space for user equipment. Tracking of the HMD and controllers provides the necessary 6DOF movement data. The positional data obtained from both the headsets internal sensors and the Base station trackers is transferred to the $\mathrm{PC}$ for processing, via separate USB cables. The appropriate multimedia data is transmitted back to the headset via HDMI. The direction of data flow and connections is illustrated in Fig4 below.

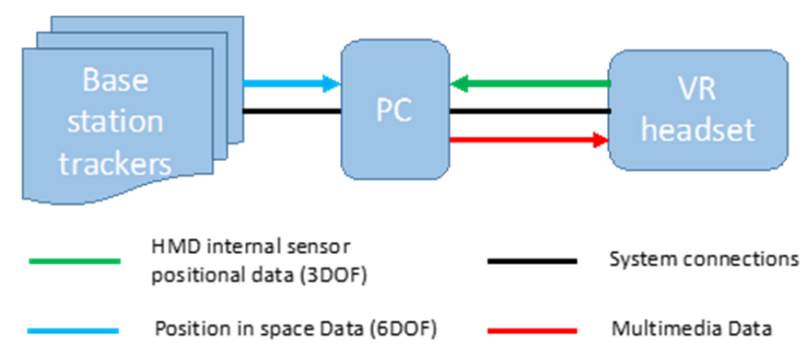

Fig. 4. General Dataflow of PCOVR system 


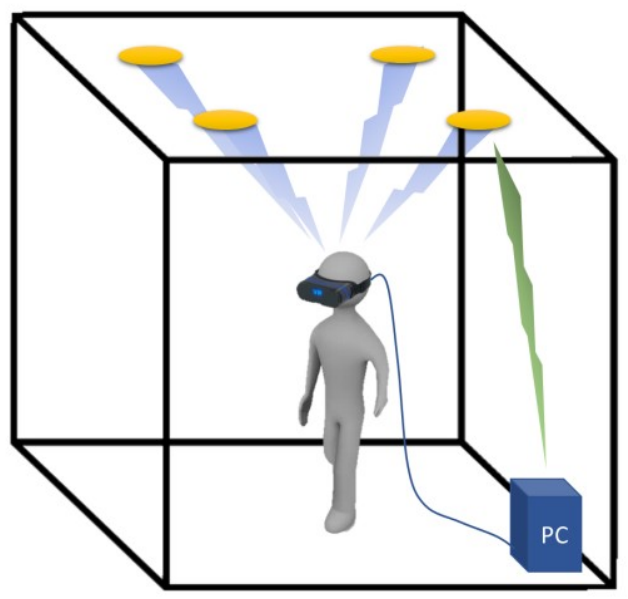

Fig. 5. IoRL IPS enabled VR concept

Fig. 5 demonstrates the first stage and initial concept of this project. The IoRL VLC and mmWave IPS tracks a dongle located on the user's HMD and thus their movements. This positional data would replace the existing external tracking system data, eliminating the need for external tracking installation and setup. The PC VR application can then request the positional data obtained from the IoRL IPS. The RRLHs can then direct this data to a second dongle connected to the computer via USB. The PC then processes the location data for the system through the internal VR application that delivers the multimedia output to the HMD via HDMI.

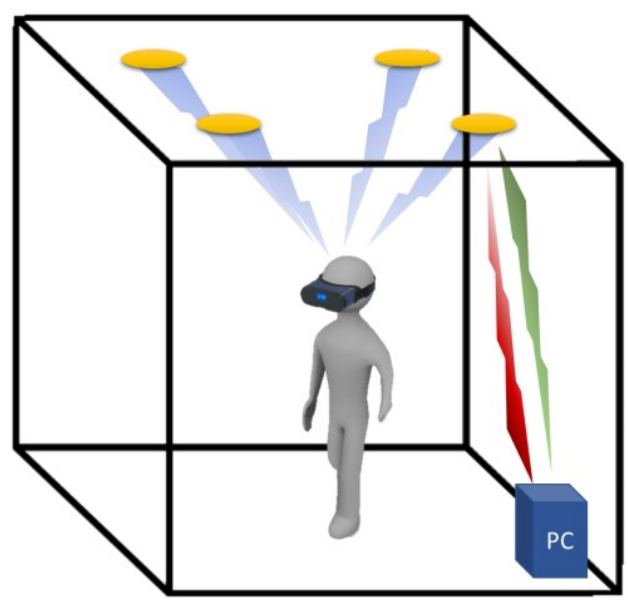

Fig. 6. IoRL wireless VR concept

Using the same system configuration, Fig. 6 shows how the IoRL RRLHs could relay information between the PC processer and the HMD. Transmitting data wirelessly with $5 \mathrm{G}$ super-fast data rates eliminates the need for wires. This multihop method involves transmitting the multimedia data output from the $\mathrm{PC}$ via the dongle to the RRLHs; the data then transmits from the RRLHs to the dongle on the HMD. In this case, the HMD dongle interfaces to the HMD via HDMI and USB to substitute the existing cables. This process is similar to the TPCAST [17] system, which uses a wireless mmWave adapter to transmit data wirelessly between the VR HMD and the computer. Fig.7. Illustrates the data flow for the proposed architecture.

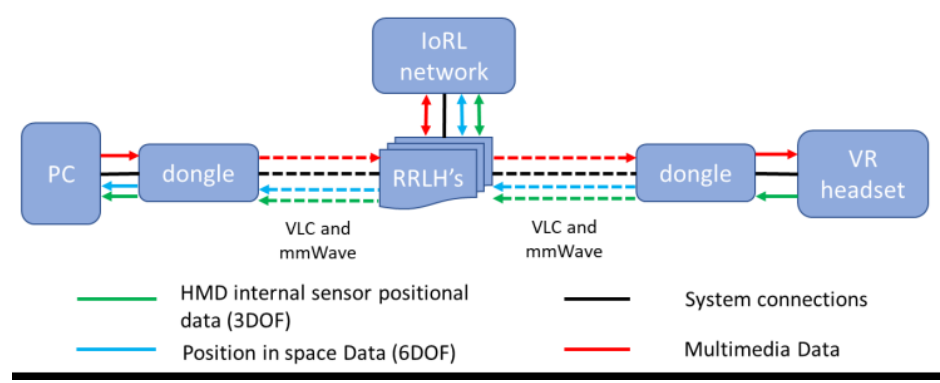

Fig. 7. Dataflow of proposed IoRL VR system

In theory, it should also be possible to reduce induced latency by transmitting the multimedia data from the PC dongle directly to the HMD dongle as an ad hoc network, however this is not within the scope of the IoRL project.

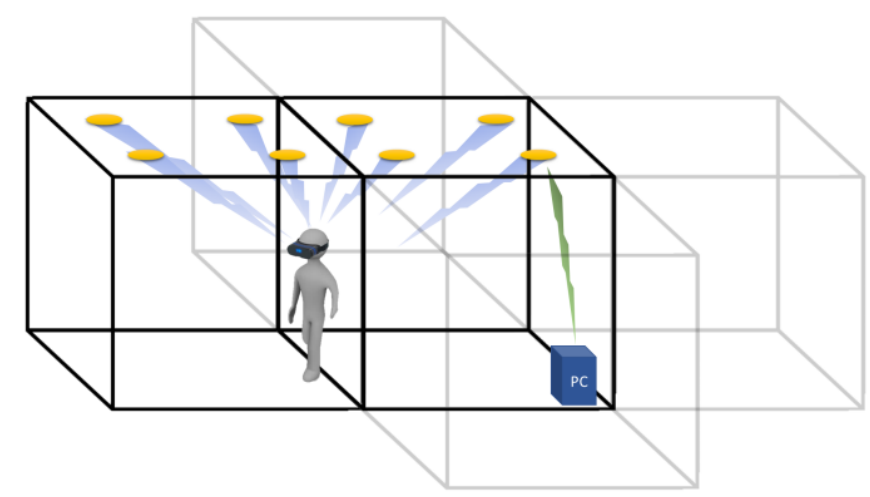

Fig. 8. IoRL Handover protocol for VR tracking between rooms

Fig. 8 depicts how by using a handover protocol, suggested by the IoRL project, for connecting UE to RRLHs between rooms, it would be possible to expand the physical explorable area. Users could easily setup a VR experience in any connected area, freely moving between rooms, opening up all available physical space.

Overall, this concept provides a safer, completely wireless, high speed, less laborious and greater PCVR experience.

\section{B. MOVR}
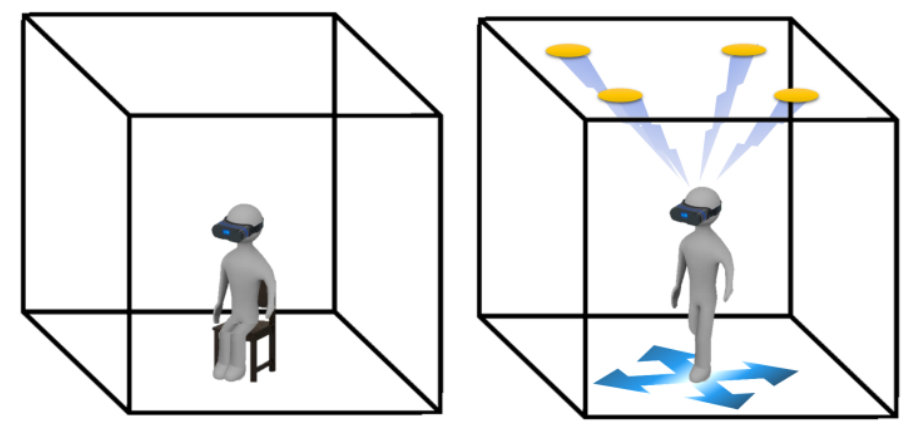

Fig. 9. Left to right respectively 9a, current MOVR and 9b, IoRL IPS enabled MOVR 
Fig. 9a illustrates the movement currently available from MOVR systems. With only 3DOF, users are limited in terms of movement and immersion. By integrating the IoRL IPS, any mobile device can be tracked via the connected dongle. This provides positional data which if requested by the mobile device could provide MOVR users the potential for $6 \mathrm{DOF}$, exploring the virtual world in a completely new way as shown in Fig. $9 b$.

VR technology is evolving rapidly and so are the potential applications. This proposed solution builds on an existing architecture (IoRL) that will be used in buildings, with potential to transform the VR experience. This solution reduces equipment cost, improves the ease of setup and permits wider use of VR. With the correct interface, this positional data could be accessed by a variety of VR operating systems, using the same tracking structure. In addition, there would be the possibility for multiple users to engage in a VR experience simultaneously given that the IoRL system tracks and provides IPS data for numerous devices.

\section{COMPARISON WITH EXISTING SOLUTIONS}

So far, wireless VR has been achieved using millimeter wave transmission between a HMD and computer, which is a similar approach to that discussed in Fig.6. This method is applied in TPCAST to deliver almost negligible induced latency. Millimeter wave transmission has also been explored for VR where simulations found it to reduce induced wireless latency substantially [18]. This demonstrates the real potential for this part of the project. In addition, standalone VR (SaVR) headsets are becoming commercially available, offering a complete all in one wireless experience.

In addition, standalone VR (SaVR) headsets are becoming commercially available, offering a complete all in one wireless experience. These HMDs require no external computer and typically incorporate some variant on inside-out technology [19]. This generally requires outward facing cameras to measure the user's movements in respect to the physical world. Headsets such as the Pico Neo [20], Oculus Project SantaCruz, Vive Focus, Qualcomm VR [21] and Lenovo mirage solo [22] all perform using the similar hardware with the exact same processor, the Snapdragon 835 [23]. This processor is commonly found in phones and thus provides a reduced level of VR when compared to PCOVR. PCOVR offers much faster processing, larger storage, higher RAM and GPU, all of which can be utilised fully by using such a proposed system. Pricing remains a challenge, the price of these commercial wireless VR systems is highly competitive. With IoRL technology still in development, the system and components are currently unique and costly. Regarding MOVR, no system yet provides 6DOF or has the potential to track remote handheld controllers, which would provide mobile phone users with much greater control in virtual environments.

\section{DEVELOPMENTS}

Low latency is a heavily contributing factor towards viable VR; this proposed approach relies greatly on providing as little induced latency as possible. Given similar low latency approaches, this remains optimistic.
While in development, the IoRL project remains a proof of concept. Though eventually it will be possible to reduce the size of the UE dongle aforementioned, the present dimensions of the dongle are too large to permit a comfortably portable system.

\section{CONCLUSION}

While there are already certain existing solutions to wireless VR and tracker-less systems none of these offer a complete, high powered solution like the above proposal does. PCOVR benefits from the increased freedom enabled by both the lack of wires as well as the ability to expand a "playable area' beyond the pre-set tracker area, all while maintaining the high processing power delivered from PCOVR. This proposal also provides an innovative solution to deliver 6DOF to MOVR. The all-in-one solution proposed within this paper would greatly improve the Virtual Reality and Augmented Reality industry, transforming how everyone uses these systems. It is worth noting however that this proposal relies greatly on the backend of the IoRL project. With the architecture still to be finalised and tested, it may be a while before the technology for this proposed system is commercially viable.

\section{ACKNOWLEGMENTS}

The authors gratefully acknowledge the European Commission for its financial support on the Horizon 2020 Internet of Radio-Light (IoRL) project No: 761992

\section{REFERENCES}

[1] John Cosmas, Yue Zhang, Xun Zhang, "Internet of Radio-Light: 5G Broadband in Buildings," European Wireless 2017, Dresden, Germany, 17th - 19th May 2017.

[2] [2] John Cosmas, Ben Meunier, Kareem Ali, Nawar Jawad, Mukhald Salih, Hong-Ying Meng et al, "5G Internet of Radio Light Services for Supermarkets" China Lighting Expo 2017, Beijing, China, 20th - 22nd November 2017.

[3] [3] Bajaj, R., Ranaweera, S. and Agrawal, D. (2002). GPS: locationtracking technology. Computer, 35(4), pp.92-94.

[4] [4] Jekabsons, G., Kairish, V. and Zuravlyov, V. (2011). An Analysis of Wi-Fi Based Indoor Positioning Accuracy. Scientific Journal of Riga Technical University. Computer Sciences, 44(1).

[5] [5] C. HUANG; X.ZHANG. VLC based Indoor Positioning System Simulation Testbed, GDR SoC-SiP Bordeaux France, 2017.

[6] [6] C. HUANG; X.ZHANG. Impact and Feasibility of Darklight LED on Indoor visible light positioning system, IEEE ICUWB SPAIN, 2017.

[7] [7] C. HUANG; X.ZHANG. LOS-NLOS idetification Algorithm for Indoor Visible Light Positioning System, 2017 20th International Symposium on Wireless Personal Multimedia Communications (WPMC)

[8] [8] Guillermo, J. and Rojas, L. (2013). Factors Influencing Virtual Reality Immersion. [online] Available at: https://s3.amazonaws.com/academia.edu.documents/35653091/VR rese arch_JohnLara.pdf?AWSAccessKeyId=AKIAIWOWYYGZ2Y53ÜL3A \&Expires $=1524483723 \&$ Signature $=$ YmvUtwbeLb4s2 WKjYliufVB5hiY $\% 3$ D\&response-content-

disposition=inline $\% 3 \mathrm{~B} \% 20$ filename $\% 3$ DFactors_Influencing_Virtual_R eality Imme.pdf

[9] [9] VR, O. (2018). Pioneering the Frontier of VR: Introducing Oculus Go, Plus Santa Cruz Updates. [online] Oculus.com. Available at: https://www.oculus.com/blog/pioneering-the-frontier-of-vr-introducingoculus-go-plus-santa-cruz-updates/ [Accessed 23 Apr. 2018]. 
[10] [10] Vive.com. (2018). VIVE Focus | HTC VIVE China. [online] Available at: https://www.vive.com/cn/product/vive-focus-en/ [Accessed 23 Apr. 2018].

[11] Larijani, L. (1994). The virtual primer. New York: McGraw-Hill.

[12] Augmented and Virtual Reality: the First Wave of 5G Killer Apps. (2018). abiresearch, Qualcomm.

[13] [12] Lang, B. (2018). An Introduction to Positional Tracking and Degrees of Freedom (DOF). [online] Road to VR. Available at: https://www.roadtovr.com/introduction-positional-tracking-degreesfreedom-dof/

[14] [13] Leadingones.com. (2018). Intro to VR: Degrees of Freedom. [online] Available at: http://www.leadingones.com/articles/intro-to-vr4.html

[15] [14] Niehorster, D., Li, L. and Lappe, M. (2017). The Accuracy and Precision of Position and Orientation Tracking in the HTC Vive Virtual Reality System for Scientific Research. i-Perception, [online] 8(3), p.2. Available http://journals.sagepub.com/doi/pdf/10.1177/2041669517708205 [Accessed 23 Apr. 2018].

[16] [15] Vive.com. (2018). VIVETM | Discover Virtual Reality Beyond Imagination. [online] Available at: https://www.vive.com/eu/

[17] [16] Wireless VR | Unleash the VR World - TPCast. (2018). Wireless VR | Unleash the VR World \&ndash; TPCast. [online] Available at: https://www.tpcastvr.com/ [Accessed 23 Apr. 2018].
[18] [17] S. Elbamby, M., Perfecto, C., Bennis, M. and Doppler, K. (2018). Edge Computing Meets Millimeter-wave Enabled VR: Paving the Way to Cutting the Cord. arXiv: $1801.07614 \mathrm{v} 3$ [cs.IT]

[19] [18] Ribo, M., Pinz, A. and Fuhrmann, A. (n.d.). A new optical tracking system for virtual and augmented reality applications. IMTC 2001. Proceedings of the 18th IEEE Instrumentation and Measurement Technology Conference. Rediscovering Measurement in the Age of Informatics (Cat. No.01CH 37188), pp.1-2.

[20] [19] Pico Interactive | VR for all - instant, easy and ultra portable. (2018). Pico Interactive | VR for all - instant, easy and ultra portable. [online] Available at: https://www.pico-interactive.com/neo [Accessed 23 Apr. 2018].

[21] [20] Qualcomm, I. (2018). Immersive Virtual Reality | Qualcomm. [online] Qualcomm. Available at: https://www.qualcomm.com/invention/cognitive-

technologies/immersive-experiences/virtual-reality [Accessed 23 Apr. 2018].

[22] [21] Lenovo Blog. (2018). Introducing the New Lenovo Mirage ${ }^{\text {TM }}$ Solo with Daydream ${ }^{\mathrm{TM}}$. [online] Available at: http://blog.lenovo.com/en/blog/cut-the-cables-and-immerse-yourself-invirtual-reality-like-never-before-wi [Accessed 23 Apr. 2018].

[23] [22] Qualcomm, S. (2018). Snapdragon 835 Mobile Platform with 10 nm 64-bit Octa Core CPU | Qualcomm. [online] Qualcomm. Available at: https://www.qualcomm.com/products/snapdragon/processors/835 [Accessed 23 Apr. 2018]. 